\title{
P86 Can Central Blood Pressure be Accurately Estimated in Individuals with and Without Systolic Blood Pressure Amplification?
}

\author{
Remi Goupil ${ }^{\star}$, Cédric Kowalski, Florence Lamarche \\ Hôpital du Sacré-Coeur de Montréal, Montreal, Canada
}

\section{ABSTRACT}

Introduction: Systolic blood pressure (SBP) does not always amplify from central to peripheral arteries. Individuals without SBP amplification (SBPamp) have higher aortic blood pressure (BP) despite similar brachial cuff SBP. To circumvent this discrepancy, the aim of this study was to determine if aortic SBP can be accurately estimated non-invasively in patients with and without SBPamp.

Methods: Patients undergoing percutaneous coronary intervention were recruited. Individuals with atrial fibrillation, $\geq 10 \mathrm{mmHg}$ between-arm SBP difference or severe aortic stenosis were excluded. Aortic and brachial intra-arterial BP were measured using a fluid-filled catheter. Simultaneously, brachial and central cuff BP were measured in triplicate (Mobil-o-Graph, IEM, Germany). Central BP was estimated by pulse wave analysis with Type I (SBP and diastolic BP) and Type II (mean and diastolic BP) calibrations. Aortic-to-brachial SBPamp was defined as $\geq 5 \mathrm{mmHg}$ increase between intra-arterial aortic and brachial SBP.

Results: Of the 151 patients recruited, only 85 had SBPamp. SBPamp+ and SBPamp- patients had similar brachial cuff SBP $(126+/-15$ vs $126+/-16 \mathrm{mmHg}, p=0.8)$ and clinical characteristics, apart from lower augmentation index in SBPamp+ $(18+/-10$ vs $22+/-11, p=0.03)$. Central BP estimated with Type I or Type II calibration could not accurately determine aortic SBPs in both phenotypes (Table 1). Using the mean of both estimates only provided a slightly better accuracy.

Conclusion: Central BP measurements cannot accurately identify the different aortic BP of the SBPamp phenotypes. A new central BP calibration may be needed to circumvent this problem.

Table 1

\begin{tabular}{lccc}
\hline Baseline characteristics & SBPamp+ $(\boldsymbol{n}=\mathbf{8 5})$ & SBPamp- $(\boldsymbol{n}=\mathbf{6 6})$ & $\boldsymbol{p}$-value \\
\hline Male sex & $74 \%$ & $74 \%$ & 1.0 \\
Age & $66 \pm 11$ & $65 \pm 9$ & 0.6 \\
Height $(\mathrm{cm})$ & $171 \pm 10$ & $170 \pm 10$ & 0.6 \\
BMI $\left(\mathrm{kg} / \mathrm{m}^{2}\right)$ & $29 \pm 6$ & $30 \pm 10$ & 0.3 \\
Active smoking & $28 \%$ & $27 \%$ & 0.9 \\
Diabetes & $19 \%$ & $17 \%$ & 0.7 \\
Hypertension & $59 \%$ & $55 \%$ & 0.6 \\
Dyslipidemia & $55 \%$ & $55 \%$ & 0.9 \\
Prior cardiovascular disease & $39 \%$ & $52 \%$ & 0.1 \\
eGFR (mL/min/1.73 $\left.\mathrm{m}^{2}\right)$ & $80 \pm 18$ & $81 \pm 18$ & 0.7 \\
Brachial cuff SBP & $126 \pm 15$ & $126 \pm 16$ & 0.8 \\
Brachial cuff diastolic blood pressure & $78 \pm 9$ & $78 \pm 12$ & 0.9 \\
Heart rate (bpm) & $67 \pm 11$ & $65 \pm 11$ & 0.3 \\
Augmentation index @ 75 bmp & $18 \pm 10$ & $22 \pm 11$ & 0.03 \\
Pulse wave velocity (m/s) & $10 \pm 2$ & $9 \pm 2$ & 0.09 \\
\hline
\end{tabular}

SBPamp+ and SBPamp- denotes individuals with and without SBP amplification, defined as $\geq 5 \mathrm{mmHg}$ increase between intra-arterial aortic and brachial SBP. Values are expressed as mean \pm standard deviation. All blood pressure measures are expressed in mmHg. $p$-values are calculated using Pearson's chi-square and Student $t$-tests. SBP, systolic blood pressure.

(c) 2019 Association for Research into Arterial Structure and Physiology. Publishing services by Atlantis Press International B.V. This is an open access article distributed under the CC BY-NC 4.0 license (http://creativecommons.org/licenses/by-nc/4.0/). 